\title{
Entanglement in the One-dimensional Kondo Necklace Model
}

\author{
A. Saguid* \\ CBPF - Centro Brasileiro de Pesquisas Físicas \\ Rua Dr. Xavier Sigaud 150 - Urca, \\ Rio de Janeiro, 22290-180, Brazil. \\ M.S. Sarandy \\ UERJ - Universidade do Estado do Rio de Janeiro \\ Rua São Francisco Xavier 524 - Maracanã, \\ Rio de Janeiro, 20550-013, Brazil.
}

(Dated: December 15, 2018)

\begin{abstract}
We discuss the thermal and magnetic entanglement in the one-dimensional Kondo necklace model. Firstly, we show how the entanglement naturally present at zero temperature is distributed among pairs of spins according to the strength of the two couplings of the chain, namely, the Kondo exchange interaction and the hopping energy. The effect of the temperature and the presence of an external magnetic field is then investigated, being discussed the adjustment of these variables in order to control the entanglement available in the system. In particular, it is indicated the existence of a critical magnetic field above which the entanglement undergoes a sharp variation, leading the ground state to a completely unentangled phase.
\end{abstract}

PACS numbers: 03.67.*,75.20.Hr,75.30.mb

\section{INTRODUCTION}

A striking feature in quantum mechanics is the presence of non-local correlations among different parts of a system. The physical property behind these purely quantum mechanical correlations is the so-called entanglement, which has been shown to be a key ingredient in quantum computation and communication [1]. Indeed, the search of experimental proposals to implement a quantum computer has strongly motivated the study of entanglement in condensed matter systems. In particular, schemes for quantum information processing based on Ising 2], XY 3], and Heisenberg [4] interactions have been suggested, leading to an intensive discussion about the thermal and magnetic entanglement in these models [5, 6, 7, 8, 9]. Furthermore, the properties of entanglement have also been analysed in other condensed matter [10, 11] and interacting quantum systems [12].

Recently, initial experiments towards the construction of quantum gates in rare-earth-ion doped crystals have been carried out [13]. The rare-earth compounds present several attractive properties for quantum computation due to the existence of a partially filled $f$-shell, which is shielded from the environment by the outer electrons. Such partially filled inner shell is a fundamental feature appearing in the heavy fermion systems 14], characterized as metallic hosts in which magnetic impurities are immersed. Thus, the discussion of the entanglement in theoretical models describing the heavy fermion physics turns out to be a subject which deserves some attention. A standard hamiltonian for this purpose is the Kondo lattice model [15, 17], which assumes the presence of one localized impurity spin on each site, coupled to the conduction electrons. From the viewpoint of

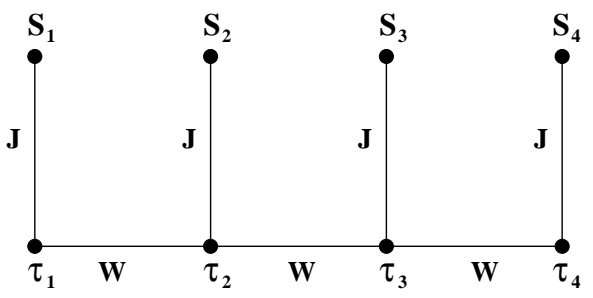

FIG. 1: Schematic arrangement of the KN chain with four sites. The spin- $1 / 2$ operators $\tau^{\alpha}$ and $S^{\alpha}$, where $\alpha=x, y, z$, denote the conduction eletrons and the spins of the local moments, respectively.

the magnetic degrees of freedom, an important feature of the model is the competition between the intra-site Kondo screening of the localized magnetic moment and the inter-site Ruderman-Kittel-Kasuya-Yosida (RKKY) interaction among the impurity spins, mediated by the conduction electrons. The Kondo coupling favors a non-magnetic ground state, while the RKKY interaction tends to establish a long range magnetic order. A scheme for entangling two conduction electrons in the presence of a magnetic impurity has been proposed by considering the Kondo hamiltonian in ref. [16].

A simplified version of the Kondo lattice hamiltonian was introduced by Doniach in ref. 17]. This model, called Kondo necklace $(\mathrm{KN})$, replaces, into the Kondo interaction, the spins of the conduction electrons by a set of pseudo-spins on a linear lattice, the charge degrees of freedom being frozen out. In spite of this approximation, the interplay between the RKKY interaction and the Kondo mechanism remains as an essential feature. The one-dimensional KN model is defined by the hamil- 
tonian

$$
H=W \sum_{i=1}^{N}\left(\tau_{i}^{x} \tau_{i+1}^{x}+\tau_{i}^{y} \tau_{i+1}^{y}\right)+J \sum_{i=1}^{N} \vec{S}_{i} \cdot \vec{\tau}_{i},
$$

where $\tau_{i}^{\alpha}$ and $S_{i}^{\alpha}$ are independent spin- $1 / 2$ operators acting on a site $i$, which are given by $\sigma^{\alpha} / 2$, with $\sigma^{\alpha}$ $(\alpha=x, y, z)$ denoting the Pauli matrices. The spin operators $\tau_{i}^{\alpha}$ and $S_{i}^{\alpha}$ are associated with the conduction electrons and the innercore spins hanging from the $\tau$ spin chain, respectively. The hamiltonian Eq. (10) describes a linear KN lattice with $N$ sites, where periodic boundary conditions are adopted, i.e., $\tau_{N+1}^{x}=\tau_{1}^{x}$ and $\tau_{N+1}^{y}=\tau_{1}^{y}$. The positive parameter $\mathrm{W}$ represents the hopping energy and $\mathrm{J}$ is the Kondo exchange coupling, which can be either ferromagnetic $(J<0)$ or antiferromagnetic $(J>0)$. A schematic arrangement of the chain is displayed in Fig. 11 Note that, since the KN model is defined with two spins on each site, the smallest representative cell of the system is described by four spins, each spin being taken as a quantum bit (qubit). A fully anisotropic version of the model, which we denote by X$\mathrm{KN}$, will also be considered. In this case, the band of the conduction electrons is represented just by an Ising term $W \sum\left(\tau_{i}^{x} \tau_{i+1}^{x}\right)$. The isotropic KN model, given in Eq. (11), will be referred from now on as XY-KN.

The aim of this work is to discuss the thermal and magnetic entanglement present in the one-dimensional KN model. The paper is organized as follows. In section II, we describe the natural thermal entanglement for both $\mathrm{XY}-\mathrm{KN}$ and X-KN models with two sites (four qubits). In section III, the entanglement in a larger chain is analysed by considering the case of four sites (eight qubits). It is observed that, qualitatively, the entanglement of the system keeps the main characteristics of the case with only two sites. Section IV is devoted to the study of the effect of a magnetic field tranversely applied to the $\tau$-chain. Finally, in section $\mathrm{V}$ we summaryze our main results and present the conclusion.

\section{THERMAL ENTANGLEMENT IN A TWO-SITE KN RING}

In this section we study the entanglement between any two qubits of the KN model with two sites at both zero and finite temperature. Before describing our results we present the definition of concurrence, which is the measure of entanglement used throughout this paper. The concurrence $C_{12}$ for a pair of qubits labelled as 1 and 2 is defined as [18]

$$
C_{12}=\max \left(\lambda_{1}-\lambda_{2}-\lambda_{3}-\lambda_{4}, 0\right),
$$

where the $\lambda_{i}$ are the square roots, in decreasing order, of the eigenvalues of the operator

$$
R \equiv \rho_{12}\left(\sigma_{y} \otimes \sigma_{y}\right) \rho_{12}^{*}\left(\sigma_{y} \otimes \sigma_{y}\right) .
$$

In Eq. (3), $\rho_{12}$ denotes the density matrix, which can be either pure or mixed, for the pair of qubits 1 and 2 , and $\rho_{12}^{*}$ its complex conjugate in the the standard basis $\{|++\rangle,|+-\rangle,|-+\rangle,|--\rangle\}$. In a system with more than two qubits, $\rho_{12}$ is obtained by tracing the density operator over the other qubits. The concurrence ranges from 0 , implying an unentangled state, to 1 , in which the two qubits are maximally entangled.

In pure states of $N$ qubits, it has been conjectured [19] that the entanglement is distributed following the inequality for the squared concurrence

$$
C_{12}^{2}+C_{13}^{2}+\ldots+C_{1 N}^{2} \leq C_{1(23 . . N)}^{2},
$$

where $C_{1(23 . . N)}$ is the single-qubit concurrence, defined as the concurrence between the qubit 1 and the rest of lattice $(23 \ldots N)$. This quantity represents the collective contributions of the entanglement between the qubit 1 and all the other qubits of the system and can be obtained from $C_{1(23 . . N)}=2 \sqrt{\operatorname{det} \rho_{1}}$, where $\rho_{1}$ is the density matrix for the qubit 1. A relevant aspect of the single-qubit concurrence is that, as it has been suggested in ref. [8], it may be a useful tool to identify a quantum critical point in a lattice system.

\section{A. XY-KN MODEL}

Let us consider the entanglement for the pairs of qubits of the two-site XY-KN model, whose spins are labelled with $\tau_{1}, \tau_{2}, S_{1}$, and $S_{2}$, as represented in Fig. 11 For simplicity, we will refer to $\tau_{1}, \tau_{2}, S_{1}$, and $S_{2}$, as qubits $A, B$, $C$, and $D$, respectively. The qubits $A$ and $B$ form a ring coupled via antiferromagnetic XY interaction $(W>0)$, while $A$ and $C$, as well as $B$ and $D$, are coupled via Heisenberg interaction, which can be either ferromagnetic or antiferromagnetic. The symmetry of the lattice ensures that the entanglement is the same for the following pairs: $A C$ and $B D, A D$ and $B C$, and $A B$ and $C D$. Therefore we will only refer to the pairs $A B, A C$, and $A D$ along the paper.

The hamiltonian for the two-site model is obtained from Eq. (1) by setting $N=2$. The ground state of the system with either $J<0$ or $J>0$ is nondegenerate and can be written as the following pure state of $A B C D$ :

$$
\begin{aligned}
|0\rangle_{x y}= & N_{x y}(|++--\rangle+|--++\rangle \\
& +\alpha_{1}(|+--+\rangle+|-++-\rangle) \\
& \left.+\alpha_{2}(|+-+-\rangle+|-+-+\rangle)\right),
\end{aligned}
$$

where the positions in the kets denote the qubits $A, B, C$, and $D$ in this order. The normalization constant $N_{x y}$ and the functions $\alpha_{1}$ and $\alpha_{2}$, which depend on the parameters $J$ and $W$, are found to be

$$
\alpha_{1}=\frac{J+2 \lambda_{x y}}{2 J}, \alpha_{2}=\frac{\lambda_{x y}^{2}+\lambda_{x y} J-3 J^{2} / 4}{W J},
$$




$$
N_{x y}=\frac{1}{\sqrt{2\left(1+\alpha_{1}^{2}+\alpha_{2}^{2}\right)}}
$$

with $\lambda_{x y}$ being the ground state energy

$$
\begin{aligned}
& \lambda_{x y}=-\frac{J}{6}-2 \sqrt{Q} \cos \frac{\theta}{3}, \\
& \theta=\arccos \left(\frac{-J\left(9 W^{2}-16 J^{2}\right)}{54 \sqrt{Q^{3}}}\right), Q=\frac{4 J^{2}+3 W^{2}}{9} .
\end{aligned}
$$

Firstly we shall discuss the natural entanglement at temperature $\mathrm{T}=0$, for which only the ground state is populated. If the $\mathrm{XY}$ coupling is taken as zero $(W=0)$, the system becomes two decoupled Heisenberg dimers, namely, $A C$ and $B D$. In this regime, all the pairs of qubits are completely unentangled for $J<0$. In fact, the absence of entanglement between qubits coupled by ferromagnetic Heisenberg interaction is a general property already pointed out in ref. [7]. On the other hand, if $J>0$ then the qubits in the dimers are maximally entangled for any value of the parameter $J$, with the concurrence vanishing for all the other pairs.

Consider now the parameter $W>0$. We obtain, in the limit $J=0$, the two-qubit antiferromagnetic XY ring in the presence of two other non-interacting spins $(C$ and $D)$. In this case, the pair of qubits $A B$ is maximally entangled for any $W$ and the concurrences for the other pairs are zero. For the Heisenberg coupling $J>0$, there is a competition, governed by the values of $J$ and $W$, between the entanglement of the pairs $A B$ and $A C$ coupled by Heisenberg and XY interactions, respectively. The concurrences $C_{A B}$ and $C_{A C}$ are shown in Figs. 2] and 3 the pair $A D$ being always unentangled. By fixing the value of $W$, it is apparent that a large value of $J$ favors the entanglement between $A C$, while small $J$ tends to increase the entanglement between the qubits $A$ and $B$. In fact, the concurrences $A B$ and $A C$ are complementary, i.e., if the qubit $A$ is partially entangled with $B$, then $A$ can have only a limited quantity of entanglement with $C$. These results satisfy completely the inequality (41), with the upper bound limit for the entanglement being obtained from the single-qubit concurrence between $A$ and $B C D$, given by $C_{A(B C D)}=1$ independently of the values of $J$ and $W$.

For the case $J<0$, the entanglement is distributed between the pairs $A B$ and $A D$, as plotted in Figs. 4 and 5 We can see that, although there is no concurrence between qubits coupled with Heisenberg interaction, an increase of the value of $J$ decreases the entanglement of the pair $A B$ and increases the concurrence for $A D$. In fact, it can be observed from Eqs. (517), that a variation in $J$ affects $C_{A B}$ and $C_{A D}$, since the functions $\alpha_{1}$ and $\alpha_{2}$ depend on this parameter. As in the case $J>0$, the inequality (4) is satisfied with $C_{A(B C D)}=1$.

In order to take into account the effect of the temperature on the entanglement in the XY-KN model, we

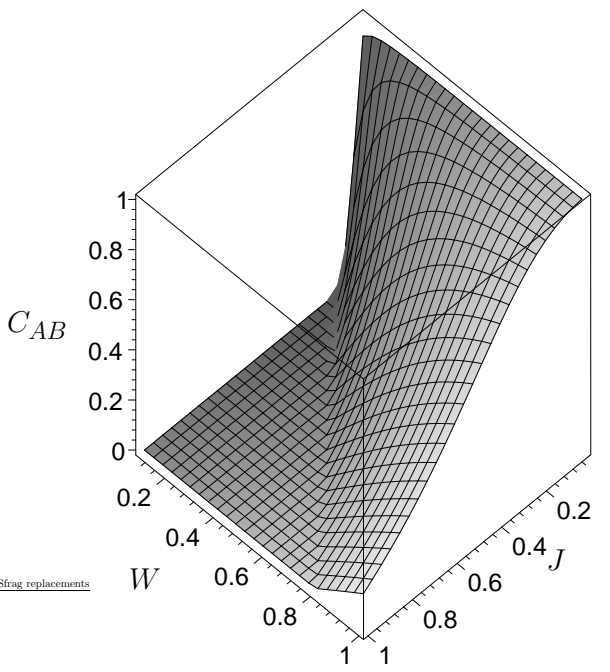

FIG. 2: Concurrence between the qubits $A$ and $B$ in the two-site XY-KN model for antiferromagnetic Heisenberg interaction at zero temperature.

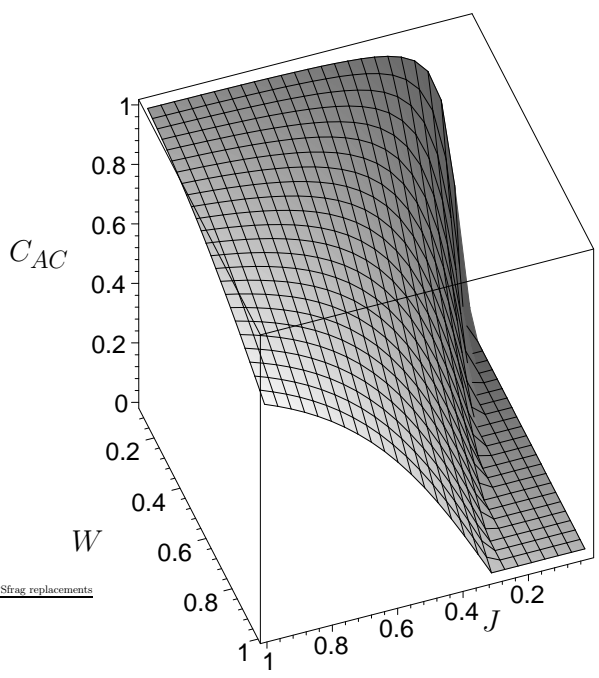

FIG. 3: Concurrence between the qubits $A$ and $C$ in the two-site XY-KN model for antiferromagnetic Heisenberg interaction at zero temperature.

consider the density matrix for the system at thermal equilibrium as given by $\rho(T)=\exp (-H / k T) / Z$, where $\mathrm{Z}$ is the partition function and $k$ the Boltzmann's constant. In Figs. [6] and 7 we show the concurrences for the pairs of qubits $A B$ and $A C$ as functions of the temperature for an antiferromagnetic Heisenberg interaction and with fixed $W=1$. By comparing the Fig. 2 to 6 and Fig. 3 to 7 we can see that, for the regime of very low temperatures, the distribution of entanglement for the pairs of qubits at $T=0$ is recovered. As the temperature increases, the entanglement for the pair $A B$ increases, for all values of $J$, before beginning to decrease. This is explained by observing that, for $J=0$, the ground state is degenerate, being a statistical mixture of four states with energy 


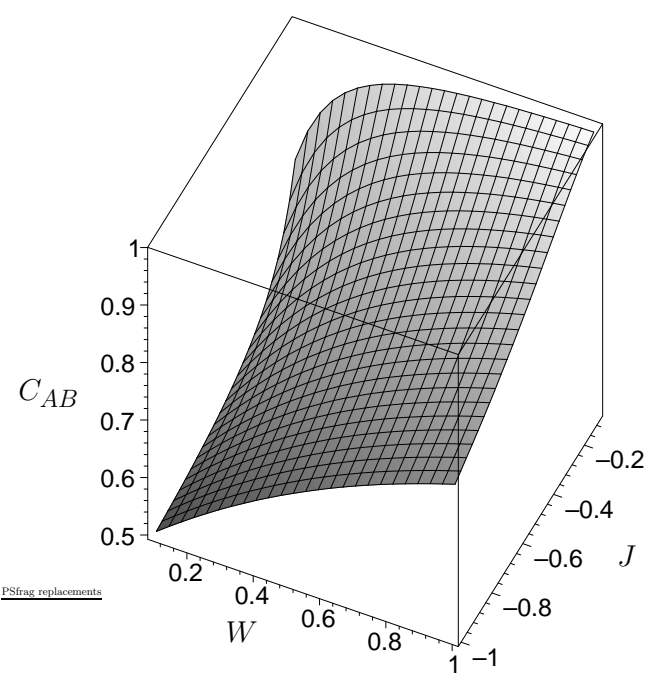

FIG. 4: Concurrence between the qubits $A$ and $B$ in the twosite XY-KN model for ferromagnetic Heisenberg interaction at zero temperature.

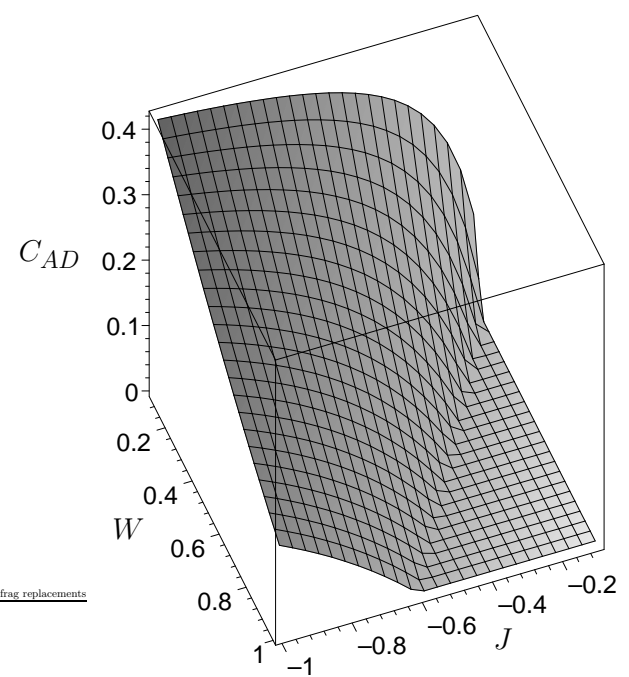

FIG. 5: Concurrence between the qubits $A$ and $D$ in the twosite XY-KN model for ferromagnetic Heisenberg interaction at zero temperature.

eigenvalue $-W$. In this case, the entanglement $A B$ assumes the maximum value 1 at $T=0$, independently of the parameter $W$. When $J$ is "turned on", the degeneracy splits up and the ground state turns out to be given by Eq. (5). However, for a nonvanishing value of the coupling $J$, there are some excited states contributing with a greater portion of entaglement $A B$ than the ground state. Thus, since the effect of the temperature is to populate the excited states, the entanglement $A B$ turns out to increase before being completely destroyed. In contrast, the entanglement for the pair $A C$ always decreases. We can understand this behavior by noting that, for $W=0$, the ground state of the system is nondegenerate and the pair $A C$ is maximally entangled for any

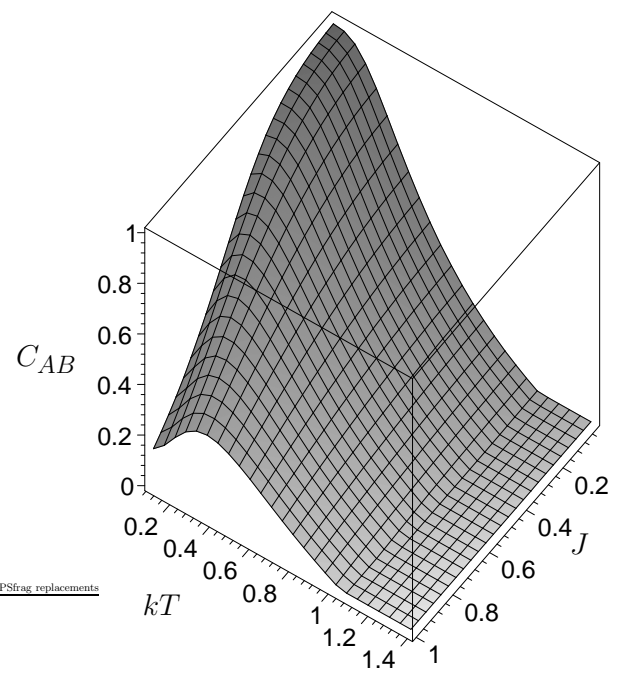

FIG. 6: Thermal concurrence in the two-site XY-KN model with antiferromagnetic Heisenberg interaction for the pair $A B$. We have set the parameter $W$ to 1 .

value of $J$ at $T=0$. For $W$ nonvanishing, the ground state of the system remains nondegenerate and there are no excited states contributing with a quantity of entanglement greater than the ground state. Therefore, the mixing of Eq. (5) with higher energy levels, in this case, can only destroy the entanglement. Moreover, as greater is the coupling $J$, greater has to be the temperature to lead $C_{A C}$ to 0 .

For ferromagnetic Heisenberg interaction, the thermal concurrence is different from zero for the pairs $A B$ and $A D$. For $A B$ it is possible to increase the entanglement with the temperature. However, the quantity of entanglement generated is very small compared to the case of the antiferromagnetic Heisenberg interaction displayed in Fig. 6

\section{B. X-KN MODEL}

In the anisotropic version of the $\mathrm{KN}$ model, the $\tau$-chain

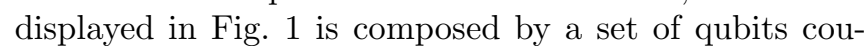
pled via Ising interactions. As it is well known [5, 8, 9], there is no entanglement naturally present for any pairs of qubits in the pure Ising chain. However, when a spin$S_{i}$ is coupled to a spin- $\tau_{i}$ of the Ising chain, by means of an antiferromagnetic Heisenberg interaction, the pair of qubits $\tau_{i} S_{i}$ becomes immediately entangled. It is worth reminding that ferromagnetic Heisenberg coupling cannot induce any concurrence in the system. In order to describe in detail the entanglement in the two-site X-KN model, let us consider four qubits $A, B, C$, and $D$, firstly at zero temperature. Similarly as defined for XY-KN model, the pair $A B$ is coupled by an Ising interaction $W>0$ and the pairs $A C$ and $B D$ by an antiferromagnetic Heisenberg interaction $J$. The ground state of the 


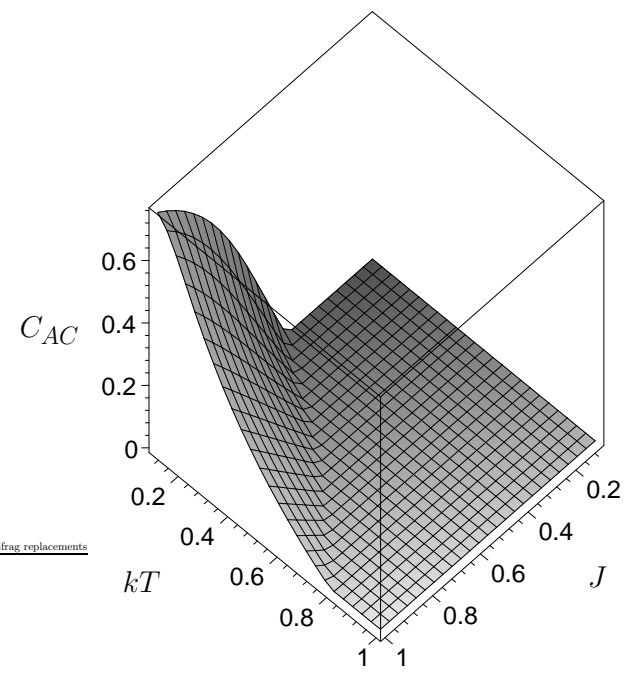

FIG. 7: Thermal concurrence in the two-site XY-KN model with antiferromagnetic Heisenberg interaction for the pair $A C$. We have set the parameter $W$ to 1 .

system can be written as

$$
\begin{aligned}
|0\rangle_{x}= & N_{x}(|++++\rangle+|----\rangle \\
& -\beta(|++--\rangle+|--++\rangle) \\
& +\beta(|+--+\rangle+|-++-\rangle) \\
& -(|+-+-\rangle+|-+-+\rangle)),
\end{aligned}
$$

where the function $\beta$ and the normalization constant $N_{x}$, which depend on the parameters $J$ and $W$, are found to be

$$
\beta=\frac{2 J+\sqrt{4 J^{2}+W^{2}}}{W}, N_{x}=\frac{1}{\sqrt{4\left(1+\beta^{2}\right)}} .
$$

From Eqs. (8) and (9), it can be shown that the entanglement for both pairs $A B$ and $A D$ is zero. The concurrence for the pair $A C$ can be expressed as

$$
C_{A C}=\frac{\beta^{2}-1}{\beta^{2}+1}
$$

reaching the maximum value in the limit $W=0$. In Fig. 8 the concurrence $C_{A C}$ is plotted as a function of the parameters $J$ and $W$. As it can be observed, although the coupling $W$ cannot induce any entanglement for the Ising coupled pairs, it contributes to decrease $C_{A C}$.

The single-qubit concurrence $C_{A(B C D)}$, between $A$ and the rest of the chain $(B C D)$, has also been computed. For $J=0$, we obtain $C_{A(B C D)}=0$ and, for any nonvanishing $J>0$, we find $C_{A(B C D)}=1$ independently of the parameter $W$. This result indicates that, in $J=0$, the system undergoes a quantum phase transition [20] characterized by a jump in the entanglement of the qubit $A$ with $B C D$, from 0 to 1 , for an infinitesimal increase of $J$. In other words, it happens a fundamental modification in

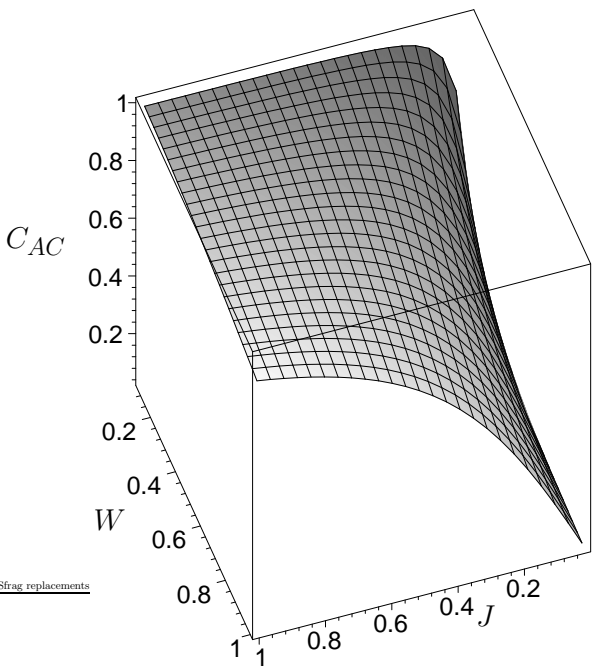

FIG. 8: The concurrence between the qubits $A$ and $C$ in the two-site X-KN model. The concurrences for the pairs $A B$ and $A D$ are zero.

the pattern of entanglement present in the ground state, which changes from a completely unentangled state to one in which the upper bound limit for the concurrence between any two qubits assumes the maximal value. This quantum phase transition is very similar to that found in ref. [5] for the transverse magnetic field Ising chain, with the coupling $J$ playing the role of the transverse field. Indeed, in the presence of random coupling constants, it has been pointed out in ref. 21] a parallelism of the critical magnetic properties between the X-KN and transverse Ising models. The results found here for the $\mathrm{X}-\mathrm{KN}$ system indicate, in the pure case (fixed $J$ and $W$ ), a similarity in the behavior of the entanglement of both models.

For completeness, we show in Fig. 9 the entanglement of the pair $A C$ as a funtion of temperature, where we have set the parameter $W=1$. We observe that the temperature tends always to decrease the entanglement.

\section{THE ENTANGLEMENT FOR A LARGER CHAIN}

In this section, we numerically describe the entaglement in the KN model with a larger number of sites. By considering the chain with four sites, as represented in Fig. 11 we show that the results found for the entanglement are qualitatively very close to that obtained earlier for a two-site X-KN and XY-KN ring. In fact, this seems to rest on the competition between the two couplings of the theory, which will take place independently of the number of sites considered.

Here we discuss the four-site XY-KN model with antiferromagnetic Heisenberg coupling at $T=0$. In this case, the concurrence is distributed among the Heisen- 


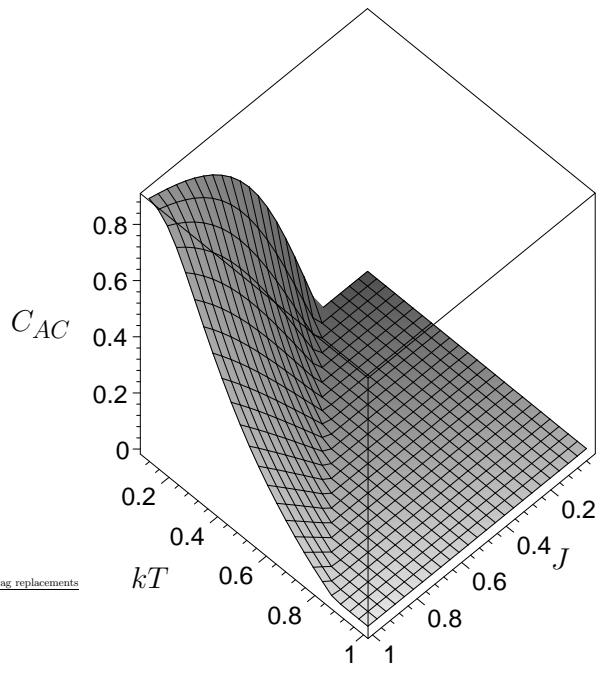

FIG. 9: Thermal concurrence for the pair of qubits $A C$ in the two-site $\mathrm{X}-\mathrm{KN}$ model. We have set the parameter $W$ to 1.

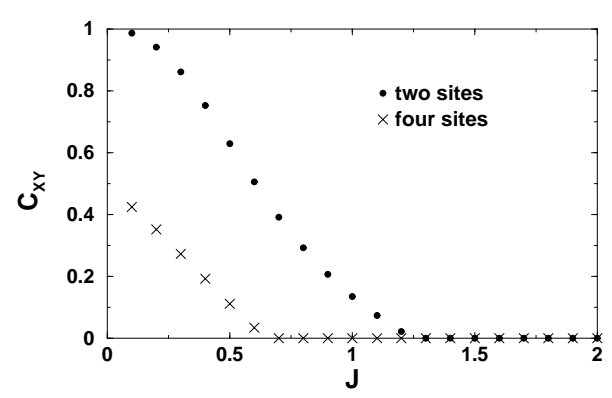

FIG. 10: Concurrence at $T=0$ for neighbor $\tau$-spins in the $\mathrm{XY}-\mathrm{KN}$ model with two and four sites. We have set $W=1$ and $J>0$.

berg coupled qubits and the neighbor and next-neighbor pairs in the $\tau$-chain. In Figs. 10 and 11] we compare the entanglement by taking the chain with two and four sites. We call $C_{X Y}$ the concurrence between neighbor spins on the $\tau$-chain, and $C_{H}$, the concurrence for neighbor spins coupled by Heisenberg interaction. We observe that, for a fixed coupling $W$, the concurrence $C_{X Y}$ decreases faster with the rise of $J$ as the number of sites becomes larger. On the other hand, $C_{H}$ is favored if many sites are taking into account, increasing faster with $J$ as we increase the size of the chain. The single-qubit concurrence remains 1 and the inequality (4) is still obeyed.

In the case of a chain with an odd number of sites we found that, due to the breaking of the ground state translational invariance 22], the entanglement between neighbor qubits on the $\tau$-chain can be different from each other. However, this difference in the behavior of odd and even number of sites is expected to disapear as the size of the chain becomes very large, as discussed for the Heisenberg model in ref. [7].

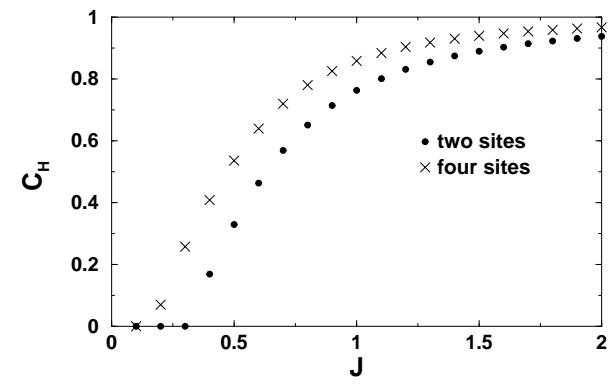

FIG. 11: Concurrence at $T=0$ for the pairs of qubits coupled by antiferromagnetic Heisenberg interaction in the XY-KN model with two and four sites. We have set $W=1$.

\section{MAGNETIC FIELD ALONG THE Z AXIS}

We investigate here how an external magnetic field applied in the KN model along the $\mathrm{z}$ direction can modify the entanglement of the system. The hamiltonian for this case is given by

$$
H_{B}=H+B \sum_{i=1}^{N}\left(s_{i}^{z}+\tau_{i}^{z}\right)
$$

where $H$ is the hamiltonian previously defined in Eq. (1D) and $B$ is a constant magnetic field.

In order to describe the magnetic entanglement in the two-site XY-KN and X-KN models, we define qubits A, $\mathrm{B}, \mathrm{C}$ and $\mathrm{D}$ in the same way as before. As discussed in section III, the qualitative results found for the two-site system hold for a larger chain, leading us to be concerned here in the former case. We first consider the magnetic entanglement in the $\mathrm{KN}$ model with antiferromagnetic Heisenberg coupling at zero temperature. For $B=0$, the ground states of the XY-KN and X-KN models are given by Eqs. (57) and (8), respectively. The single-qubit concurrence in both cases is always 1 , independently of the values of the parameters $J$ and $W$. After the external magnetic field $B$ is "turned on", the ground state of the $\mathrm{XY}-\mathrm{KN}$ model suddenly changes, as $B$ crosses a specific value, from Eq. (5) to

$$
\begin{aligned}
&|0\rangle_{x y}^{B}=N_{x y}^{B}( \gamma_{1}(|---+\rangle-|--+-\rangle) \\
&\left.-\gamma_{2}(|-+--\rangle+|+---\rangle)\right)
\end{aligned}
$$

where $N_{x y}^{B}$ is the normalization constant and the $\gamma_{i}$ are functions of the parameters $J$ and $W$. In this state the single-qubit concurrence has a little decrease from 1 to a value which depends on $J$ and $W$. For example, when $J=W=1$, the value of the single-qubit concurrence is 0.989. By increasing the magnetic field to a critical value $B_{c}=B_{c}(J, W)$ the single-qubit concurrence vanishes, since the ground state becomes the fully unentangled state $|----\rangle$. Hence, for any fixed value of the 


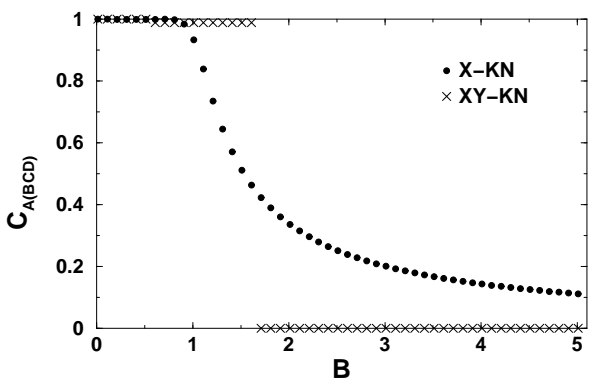

FIG. 12: The single-qubit concurrence for both two-site XY$\mathrm{KN}$ and $\mathrm{X}-\mathrm{KN}$ models with antiferromagnetic Heisenberg interaction at $\mathrm{T}=0$. The parameters $J$ and $W$ are set to 1 . For the XY-KN model the system presents a quantum phase transition at $B_{c} \approx 1.707$. For the $\mathrm{X}-\mathrm{KN}$ model the concurrence decays analytically with $B$.

parameter $W$, we obtain a critical line $B_{c}=B_{c}(J)$. Taking $J=W$, we can numerically interporlate the straight line $B_{c} \approx 1.707 \mathrm{~J}$. We observe that the effect of the external magnetic field is the gradual alignement of all the spins in the chain, leading the ground state always to an unentangled state for a high value of $B$.

The behavior for the X-KN model in the presence of a magnetic field is quite different to that of the XYKN model. When we "turn on" the magnetic field, the ground state given by Eq. (8) generalizes to

$$
\begin{aligned}
|0\rangle_{x}^{B}=N_{x}^{B} & \left(\epsilon_{1}|++++\rangle+\epsilon_{2}|----\rangle\right. \\
& +\epsilon_{3}|++--\rangle+\epsilon_{4}|--++\rangle \\
& +\epsilon_{5}(|+--+\rangle+|-++-\rangle) \\
& \left.+\epsilon_{6}(|+-+-\rangle+|-+-+\rangle)\right),
\end{aligned}
$$

where $N_{x}^{B}$ is the normalization constant and the $\epsilon_{i}$ are functions of the parameters $J$ and $W$. By increasing $B$, all the functions $\epsilon_{i}$ decrease except $\epsilon_{2}$, which becomes higher. In the limit $B \rightarrow \infty$ the ground state becomes the unentangled state $|----\rangle$. In Fig. 12] it is exhibited the single-qubit concurrence for the XY-KN and X-KN models at zero temperature, with the parameters $J$ and $W$ set to one. As it can be seen, the single-qubit concurrence is an analytical function of the magnetic field $B$ for the X-KN model, showing a smooth decrease of the entanglement of the ground state. This behavior is clearly different from the XY-KN case, in which the concurrence undergoes a sharp variation.

Let us now consider the thermal entanglement in the model by taking into account the presence of the magnetic field $B$. As discussed in Section $I I A$ for the XY-KN chain with antiferromagentic Heisenberg interaction, the increase of the temperature can rise the entanglement of $A B$ due to the mixing of the ground state with some higher entangled excited states. In contrast, the concurrence for the pair $A C$ is always decreased as the temper-

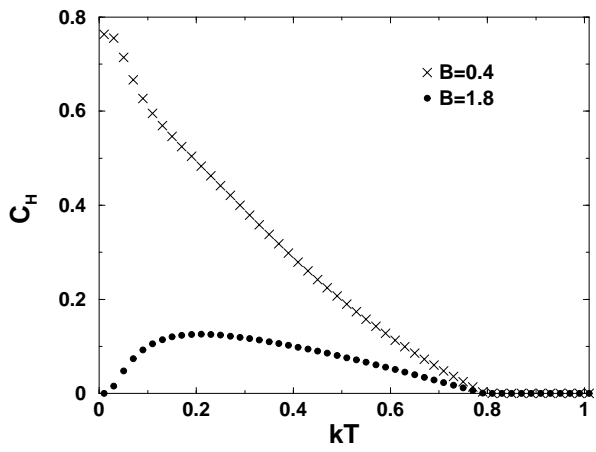

FIG. 13: Thermal concurrence for the pairs of qubits coupled by antiferromagnetic Heisenberg interaction in the two-site XY-KN model. The parameters $J$ and $W$ are set to 1 . As $B$ crosses the critical value $B_{c} \approx 1.707$ the concurrence increases with the temperature before vanishing at $T \approx 0.79$.

ature is "turned on". However, when a magnetic field $B$ greater than the critical value $B_{c}$ is applied in the system, the concurrence for the pair $A C$ can considerably be increased as the temperature rises, as exhibited in Fig. 13. This is because, as $B$ crosses $B_{c}$, the ground state of the two-site XY-KN model suddenly changes from a highly entangled to an unentangled state. Hence, the increase of the temperature can here generate entanglement also for the pair $A C$ due to the mixing of the unentangled ground state with some entangled excited states. For $B>B_{c}$, as $B$ approaches the critical field $B_{c}$, the entanglement becomes higher with the increase of the temperature. Moreover numerical simulations point to the existence of a temperature for which the entanglement is always destroyed indenpendently of the magnetic field applied. For $J=W=1$, this temperature is $T_{c} \approx 0.79$ when we compute the concurrence for a Heseinberg coupled pair.

We have also studied the behavior of the concurrence in the case of ferromagnetic Heisenberg coupling in the presence of the transverse magnetic field. The results found for the single-qubit concurrence are qualitatively the same as the antiferromagnetic case in both X-KN and $\mathrm{XY}-\mathrm{KN}$ models, the difference being that now a smaller value of $B$ is necessary for destroying the entanglement.

\section{CONCLUSION}

In this paper we have discussed the thermal and magnetic entanglement in the one-dimensional KN hamiltonian, which is a simple model for describing heavy fermion systems. Concerning the natural entanglement present in the model, we have found that the concurrence for a pair of qubits is distributed according to the fundamental couplings of theory. Furthermore we have been able to indicate, in the fully anisotropic version of the system, i.e. the $\mathrm{X}-\mathrm{KN}$ model, a quantum phase transi- 
tion for the Heisenberg interaction $J=0$. The critical behavior of the entanglement has been characterized by means of the single-qubit concurrence, which is the upper bound limit for the entanglement present in the chain. The effect of the temperature and the presence of an external magnetic field applied in the $\mathrm{z}$ direction has also been studied. These variables have been shown to be adjustable to control the entanglement available in the system. In particular, it has been found, in the XY-KN model, a critical magnetic field $B_{c}(J, W)$ which separates phases exhibiting a completely different pattern of entanglement.

It is known the KN model presents a rich phase diagram with remarkable critical magnetic properties [23]. It would be interesting to search a connection of this critical behavior with the results found in this paper. Efforts in this direction would contribute to a more complete understanding of the relationship between entanglement and quantum phase transitions in condensed matter systems as originally proposed in refs. [8, 9].

\section{ACKNOWLEDGEMENTS}

We would like to thank Conselho Nacional de Desenvolvimento Científico e Tecnológico-CNPq Brasil for financial support.

* Electronic address: asaguia@cbpf.br

$\dagger$ Electronic address: sarandy@dft.if.uerj.br

[1] M.A. Nielsen and I.L. Chuang, Quantum Computation and Quantum Information, Cambridge University Press, 2000

J. Preskill, Physics 229 (1998), Lecture notes on-line at http://www.theory.caltech/edu/people/preskill/ph229/

[2] B.E. Kane, Nature 393, 133 (1998).

[3] A. Imamog̃lu, D.D. Awschalom, G. Burkard, D.P. DiVincenzo, D. Loss, M. Sherwin, and A. Small, Phys. Rev. Lett. 83, 4204 (1999).
[4] G. Burkard, D. Loss, and D.P. DiVincenzo, Phys. Rev. B 59, 2070 (1999).

[5] D. Gunlycke, V.M. Kendon, V. Vedral, and S. Bose, Phys. Rev. A 64, 042302 (2001).

[6] X. Wang, Phys. Rev. A 64, 012313 (2001).

[7] M.C. Arnesen, S. Bose, and V. Vedral, Phys. Rev. Lett. 87, 017901 (2001).

[8] T.J. Osborne and M.A. Nielsen, e-print quant-ph/0109024 Phys. Rev. A 66, 032110 (2002).

[9] A. Osterloh, L. Amico, G. Falci, and R. Fazio, Nature 416, 608 (2002).

[10] M.A. Martín-Delgado, e-print quant-ph/0207026

[11] I. Bose and E. Chattopadhyay, e-print cond-mat/0208011

[12] K. Audenaert, J. Eisert, M.B. Plenio, and R.F. Werner, Phys. Rev. A 66, 042327 (2002).

[13] N. Ohlsson, R.K. Mohan, and S. Kröll, Opt. Communications 201 (2001) 71;

M. Nilsson, L. Levin, N. Ohlsson, T. Christiansson, and S. Kröll, e-print quant-ph/0201141

[14] M.A. Continentino, Quantum Scaling in Many-Body Systems, World Scientific, 2001;

A.C. Hewson, The Kondo Problem to Heavy Fermions, Cambridge University Press, 1993.

[15] T. Kasuya, Prog. Theor. Phys. 16, 45 (1956); S. Doniach, Valence Instabilities and Narrow Band Phenomena, Edited by R. Parks, 34, Plenum, 1977.

[16] A.T. Costa Jr. and S. Bose, Phys. Rev. Lett. 87, 277901 (2001).

[17] S. Doniach, Physica 91B, 231 (1977).

[18] S. Hill and W.K. Wootters, Phys. Rev. Lett. 78, 5022 (1997); W.K. Wootters, Phys. Rev. Lett. 80, 2245 (1998).

[19] V. Coffman, J. Kundu, and W.K. Wootters, Phys. Rev. A 61, 052306 (2000).

[20] S. Sachdev, Quantum Phase Transitions, Cambridge University Press, 1999.

[21] T.G. Rappoport, B. Boechat, M.A. Continentino, and A. Saguia, e-print cond-mat/0111123

[22] K.M. O'Connor and W.K. Wootters, Phys. Rev. A 63, 052302 (2001).

[23] T.G. Rappoport, A. Saguia, B. Boechat, and M.A. Continentino, Phys. Rev. B 64, 140402(R) (2001); G.M. Zhang, Q. Gu, and, L. Yu, Phys. Rev. B 62, 69 (2000). 